\title{
ARTÍCULO ORJGINAL
}

\section{Fuentes-Valera SR ${ }^{1}$ \\ Mejía-Serván PB ${ }^{1}$ \\ Mondragón-Vega LA' \\ Valera-Montoya IS ${ }^{1}$ \\ Zegarra-Domínguez CT$^{1}$ \\ Arriola-Guillén LE ${ }^{2}$}

1 Residentes de la Especialidad de Ortodoncia, Escuela de Estomatología, Universidad Científica del Sur

2 Docente principal de la Especialidad de Ortodoncia, Escuela de Estomatología, Universidad Científica del Sur

\section{VERTICALIZACIÓN DE MOLARES: UNA REVISIÓN DE CASOS CLÍNICOS DE LOS DIFERENTES MÉTODOS UTILIZADOS}

\section{RESUMEN}

Uno de los desafíos que enfrenta el ortodoncista es la mecánica de verticalización de molares, ya sea por la pérdida prematura de piezas posteriores, impactación de segundas, o terceras molares que servirían para reemplazar a sus antecesoras. Existen diferentes estudios que muestran distintas formas de lograr la verticalización. El propósito de esta revisión de casos fue evaluar los métodos utilizados para la verticalización de molares inferiores, tiempo de acción y los efectos indeseados, publicados en la literatura científica. Se realizó una busqueda electrónica de la literatura médico-dental en la librería científica Cochrane, PubMed, Scielo y Medline así como en las revistas especializadas del área de Ortodoncia desde el año 1970 al 2016 usando las palabras clave: Molar uprighting, miniscrew, cantilever. Se encontraron 47 artículos relacionados a verticalización de molares inferiores. Después de la selección basada en los criterios de elegibilidad, 9 estudios, calificaron para esta revisión de casos. Se realizó también una evaluación de la calidad de los artículos incluidos. Se observó que el tipo de anclaje más eficiente fueron los microtornillos, ya que no producen efectos colaterales en piezas anteriores y permiten la verticalización de la molar sin extrusión. Sin embargo, se necesitan estudios comparativos de alta validez de las diferentes técnicas de verticalización de molares en los cuales se evalúen los efectos indeseados y el tiempo de obtención de resultados, para presentar una sólida evidencia como sustento de los resultados de esta revisión de casos clínicos.

PALABRAS CLAVE: Verticalización molar, minitornillos, cantilever.

MOLAR VERTICALIZATION: A REVIEW OF CLINICAL CASES OF THE DIFFERENT METHODS USED ABSTRACT

One of the challenges the orthodontist deal with, is the mechanics of molars uprighting, either by the premature loss of posterior teeth, impaction of the second or third molars that serve to replace their predecessors. Different studies show several ways to achieve molar uprighting. The purpose of this review was to evaluate methods used for upright lower molars, action time and unwanted effects, published in the scientific literature. An electronic search of the medical and dental literature in Cochrane, PubMed, Scielo and Medline scientific library and specialized journals in the field of Orthodontics from 1970 to 2016 was performed using keywords: molar uprighting, miniscrew, cantilever was performed. 47 articles related to lower molars uprighting were found. After selection based on the eligibility criteria, nine studies qualified for the review of cases. Quality assessment of the included articles was also performed. It was observed that the most efficient type of anchorage are miniscrews, it does not produce side effects in anterior teeth and allows uprighting of the target molar without extrusion. However, high validation comparative studies of different molar uprighting techniques are needed, in which the unwanted effects and the time of obtaining results are evaluated, to present solid evidence as support of the results of this review of clinical cases. 


\section{INTRODUCCIÓN}

En la actualidad uno de los desafíos que enfrenta el ortodoncista en su práctica cotidiana es la mecánica de verticalización de molares, ya sea por la pérdida prematura de piezas posteriores como primeras o segundas molares, impactación de terceras molares que servirían para reemplazar a sus antecesoras o pacientes derivados por parte del rehabilitador que presentan espacios edéntulos posteriores con mesioinclinación de las piezas adyacentes. El manejo por parte del ortodoncista involucra la verticalización de estas piezas para así permitir una mejor rehabilitación de la zona edéntula logrando de esta manera una mejor distribución de las cargas oclusales para su futura rehabilitación o el cierre de espacios. ${ }^{1}$

Para lograr la verticalización de las piezas existen actualmente diferentes métodos; entre ellos se encuentran, los resortes de niquel titanio con o sin anclaje en minitornillos, así como también los brazos de palanca y cantilever. Estos métodos dependen de la destreza del operador, la biomecánica, la salud periodontal del paciente, entre otros. $^{2-4,9-11}$

Hasta el momento, no se dispone de ninguna revisión sistemática respecto a esta situación clínica, ni metaanálisis, tampoco ensayos clínicos aleatorizados, ni trabajos de cohorte, encontrándose sólo un estudio retrospectivo con 125 casos; ${ }^{1}$ la mayoría de investigaciones con respecto a la verticalización de molares son reportes de máximo tres casos que describen mecanismos de verticalización, tiempo de tratamiento, biomecánica, confort del paciente, descripción del anclaje, cantidad de verticalización lograda y efectos indeseados..$^{2-4,9-11}$

Siendo así, el propósito de esta revisión de casos clínicos fue evaluar los métodos utilizados para la verticalización de molares inferiores, tiempo de acción y los efectos indeseados, publicados en la literatura científica. Para lograr responder las siguientes preguntas.
- ¿Cuál es el método más efectivo y eficiente para la verticalización de piezas posteriores?

- ¿Qué método logra la verticalización en el menor tiempo?

- ¿Qué tipo de anclaje es el más eficiente?

\section{MATERIALES Y MÉTODOS}

Esta investigación comenzó siguiendo los parámetros del PRISMA checklist para las revisiones sistemáticas de ciencias de la salud. Debido a que no se encontraron estudios longitudinales de verticalización de molares mandibulares ni ensayos clínicos aleatorizados, se incluyeron los reportes de casos de las diferentes fuentes de información disponibles. Una búsqueda de la literatura medico-dental en la librería científica Cochrane, PubMed, Scielo y Medline así como en las revistas especializadas del área de Ortodoncia desde el año 1970 al 2016 usando las palabras claves, molar uprighting, miniscrew, cantilever. (Tabla 1)

\section{Criterios de Elegibilidad}

Al no encontrar revisiones sistemáticas, ensayos clínicos ni estudios de seguimiento, se decidió incluir reportes de caso, con por lo menos dos situaciones clínicas. Se encontraron 47 artículos relacionados a verticalización de molares inferiores, independientemente si eran con la misma técnica de verticalización o no.

\section{Los criterios de inclusión de los artículos fue- ron:}

- Reporte de casos en humanos.

- Estudios de verticalización de molares inferiores.

- Verticalización de una sola pieza.

\section{Los criterios de exclusión fueron:}

- Tesis, cartas, editoriales, reportes de casos donde no aparecía el abstract.

- Estudios de animales.

- Verticalización de molares superiores. 
TABLA 1

\section{ARTÍCULOS SELECCIONADOS DE DIFERENTES FUENTES DE INFORMACIÓN DE ORTODONCIA}

\begin{tabular}{|c|c|c|c|c|}
\hline Revista & $\begin{array}{c}\text { Número de } \\
\text { Artículos } \\
\text { encontrados }\end{array}$ & $\begin{array}{l}\text { Artículos } \\
\text { que no } \\
\text { cumplieron } \\
\text { criterios de } \\
\text { selección }\end{array}$ & $\begin{array}{l}\text { Artículos } \\
\text { que } \\
\text { cumplieron } \\
\text { criterios de } \\
\text { selección }\end{array}$ & Web \\
\hline AJO-DO & 12 & 10 & 2 & http://www.ajodo.org/ \\
\hline $\begin{array}{l}\text { The Angle of } \\
\text { Orthodontist }\end{array}$ & 5 & 4 & 1 & http://www.angle.org/ \\
\hline Dental Press & 6 & 5 & 1 & http://dpjo.dentalpresspub.com/ \\
\hline EJO & 1 & 1 & - & http://ejo.oxfordjournals.org/ \\
\hline $\begin{array}{l}\text { Journal World } \\
\text { Federation }\end{array}$ & 1 & 1 & - & http://www.jwfo.org/ \\
\hline $\mathrm{JCO}$ & 11 & 6 & 5 & https://www.jco-online.com/ \\
\hline JADA & 2 & 2 & - & http://jada.ada.org/ \\
\hline $\begin{array}{l}\text { Journal of } \\
\text { Prosthetic } \\
\text { Dentistry }\end{array}$ & 2 & 2 & - & http://www.thejpd.org/ \\
\hline $\begin{array}{l}\text { Journal of } \\
\text { Prosthodontic } \\
\text { Research }\end{array}$ & 1 & 1 & - & $\begin{array}{l}\text { http://www. } \\
\text { journalofprosthodonticresearch. } \\
\text { com/ }\end{array}$ \\
\hline $\begin{array}{l}\text { Revista de la U } \\
\text { Sao Paulo }\end{array}$ & 1 & 1 & - & http://www.usp.br/revistausp/ \\
\hline $\begin{array}{l}\text { Progress in Or- } \\
\text { thodontics }\end{array}$ & 1 & 1 & - & $\begin{array}{l}\text { http://www.progressinorthodontics. } \\
\text { com/ }\end{array}$ \\
\hline Total & 43 & 34 & 9 & \\
\hline
\end{tabular}

\section{Recolección de datos:}

Se realizó una búsqueda exhaustiva de la literatura científica, para identificar todos los estudios que evaluaron la verticalización de molares inferiores, su biomecánica y tiempo de tratamiento. Sólo se incluyeron los reportes con no menos de dos casos. La edad y el género no se consideraron como restricción.

Los datos fueron recolectados y analizados de acuerdo a los siguientes puntos:
- Revista en la que se publicó.

- Diseño del estudio.

- Tamaño de muestra.

- Tipo de mecánica utilizada.

- Tipo de anclaje.

Finalmente, sólo nueve artículos fueron revisados para evaluar los diferentes métodos de verticalización de molares. 


\section{Capacitación:}

Un equipo de revisión de cinco personas (SRFV, los investigadores para definir la validez de los PBMS, LAMV, ISVM, CTZD) realizaron una estudios recolectados, se utilizaron 4 criterios búsqueda individual de los artículos en los buscadores mencionados, se evaluaron los títulos y resúmenes de los documentos en una reunión de (Tabla 2-4).

\section{TABLA 2}

REQUERIMIENTOS EVALUADOS POR LOS INVESTIGADORES DE LOS ARTÍCULOS OBTENIDOS DESPÚES DE LOS PRIMEROS FILTROS

\begin{tabular}{|l|c|c|c|c|c|c|c|c|c|c|}
\hline & \multicolumn{7}{|c|}{ Artículos } & & & \\
\hline Criterio & 1 & 2 & 3 & 4 & 5 & 6 & 7 & 8 & 9 & Promedio \\
\hline $\begin{array}{l}\text { Descripción } \\
\text { detallada del } \\
\text { procedimiento }\end{array}$ & 4 & 4 & 4 & 3 & 4 & 4 & 4 & 3 & 4 & 3 \\
\hline Número de casos & 4 & 2 & 3 & 2 & 3 & 2 & 3 & 3 & 3 & 4 \\
\hline $\begin{array}{l}\text { Eficacia del } \\
\text { tratamiento }\end{array}$ & 3 & 4 & 4 & 4 & 4 & 3 & 4 & 3 & 4 & 4 \\
\hline $\begin{array}{l}\text { Tiempo de } \\
\text { tratamiento }\end{array}$ & 4 & 3 & 4 & 3 & 3 & 3 & 0 & 3 & 3 & 4 \\
\hline Total: & 15 & 13 & 15 & 12 & 14 & 12 & 11 & 12 & 14 & \\
\hline
\end{tabular}

La identificación del número del artículo se observa en el cuadro de descripción de características y resultados de la investigación.

TABLA 3

\section{VALORES NUMÉRICOS PARA DETERMINAR LA CALIDAD DE CADA CRITERIO EVALUADO EN CADA ESTUDIO}

\begin{tabular}{|l|c|}
\hline CALIDAD DE CADA CRITERIO & Valor \\
\hline Muy Bueno & 4 \\
\hline Bueno & 3 \\
\hline Medio & 2 \\
\hline Malo & 1 \\
\hline Muy Malo & 0 \\
\hline
\end{tabular}

TABLA 4

VALORES NUMÉRICOS PARA DETERMINAR LA CALIDAD DE CADA ESTUDIO

\begin{tabular}{|l|c|}
\hline CALIDAD DEL ESTUDIO & Valor \\
\hline Muy Bueno & 16 \\
\hline Bueno & $12-15$ \\
\hline Medio & $8-11$ \\
\hline Malo & $4-7$ \\
\hline Muy Malo & $0-3$ \\
\hline
\end{tabular}




\section{RESULTADOS}

La estrategia de búsqueda produjo 47 artículos. Después de la selección y basándose en los criterios de elegibilidad, nueve artículos calificaron para el análisis final (Tabla 1). Los textos completos de estos documentos fueron obtenidos para el análisis.

\section{Calidad de los estúdios:}

- La calidad de investigación y consistencia metodológica fue buena en ocho estudios, y en uno media.

- El estudio que obtuvo calificación más alta fue un estudio retrospectivo que incluyó la verticalización de 125 molares inferiores. ${ }^{1}$

Se observó que el método más efectivo y eficiente para la verticalización de piezas posteriores es el uso de minitornillos en combinación con resorte abierto, alambre flexible, cadena de poder o hilo elastomérico, ya que no produce efectos colaterales en piezas anteriores o adyacentes y permite la verticalización de la pieza sin extrusión de ésta, además de lograrlo en un periodo de tiempo promedio de cinco a seis meses.

El método que logra la verticalización en el menor tiempo es el del brazo de palanca confeccionado en TMA 0.016 " x 0.022 ", sujeto a piezas adyacentes a la molar a verticalizar, con un tiempo de 2 a 4 meses, pero no fueron evaluados los efectos no deseados en las piezas de anclaje.

El tipo de anclaje más eficiente es el de minitornillos, ya que no produce efectos colaterales en piezas anteriores y permite la verticalización de la pieza sin extrusión.(Figuras 1-9)
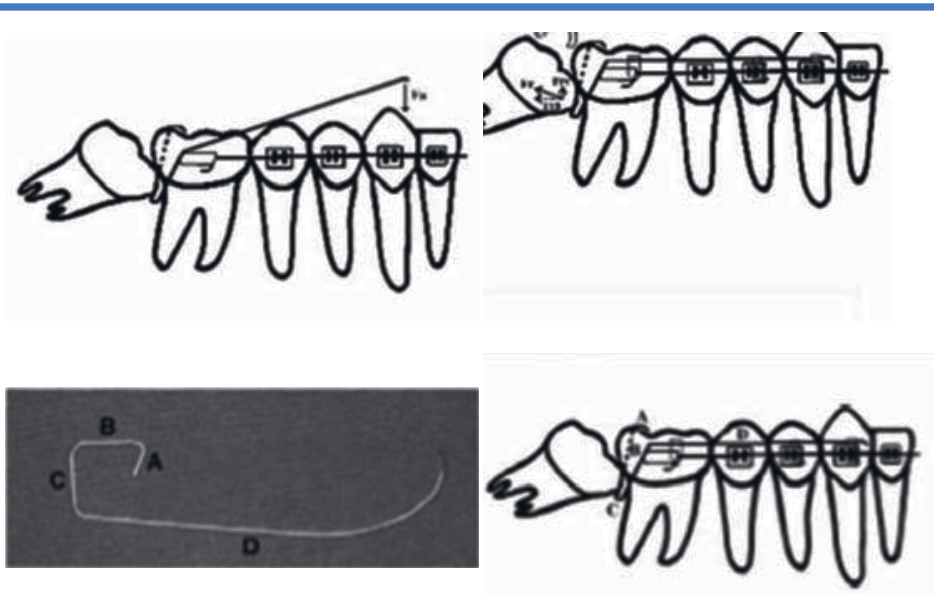

Figura 1. Fu et al. $(2012)^{1}$

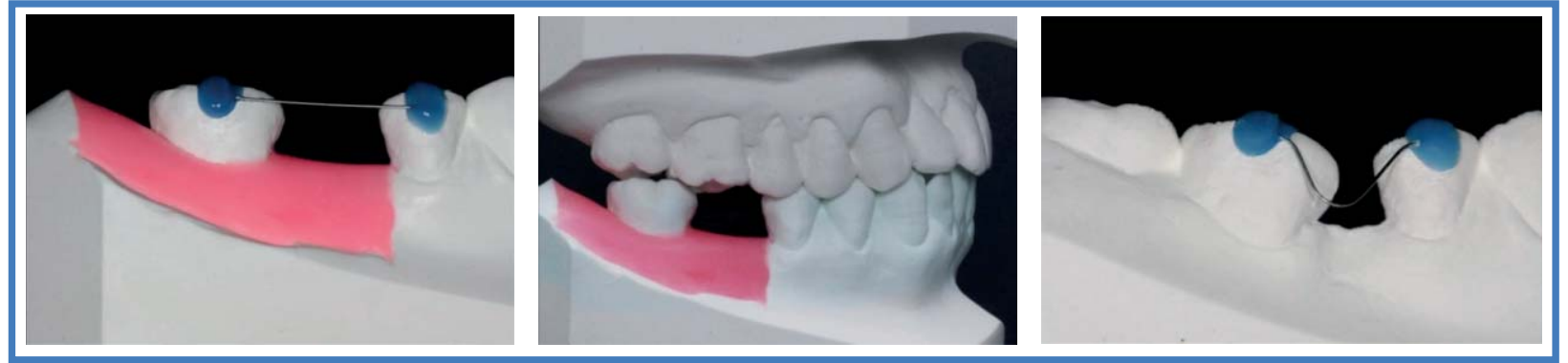

Figura 2. Kim et al. $(2014)^{2}$ 


\begin{tabular}{|c|c|c|}
\hline 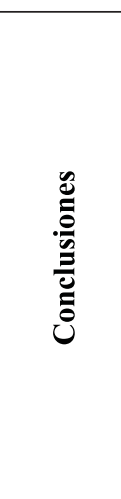 & 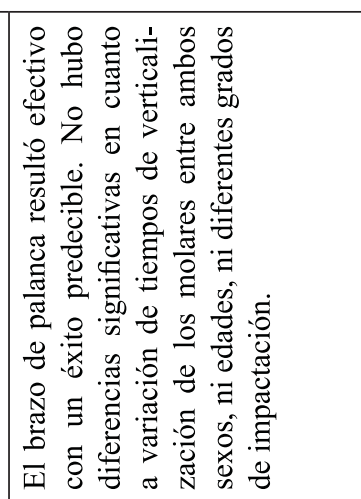 & 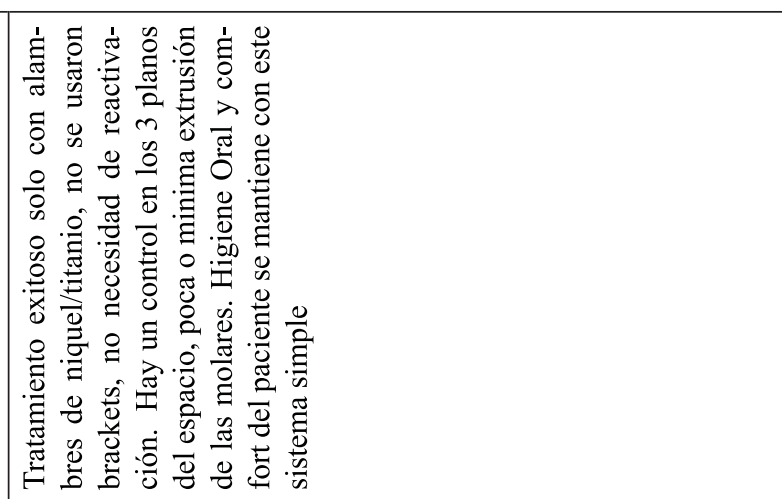 \\
\hline 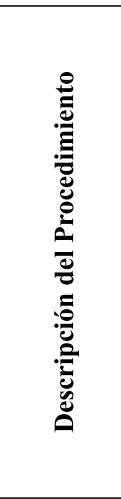 & 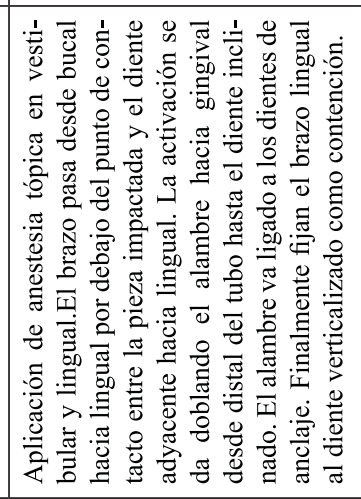 & 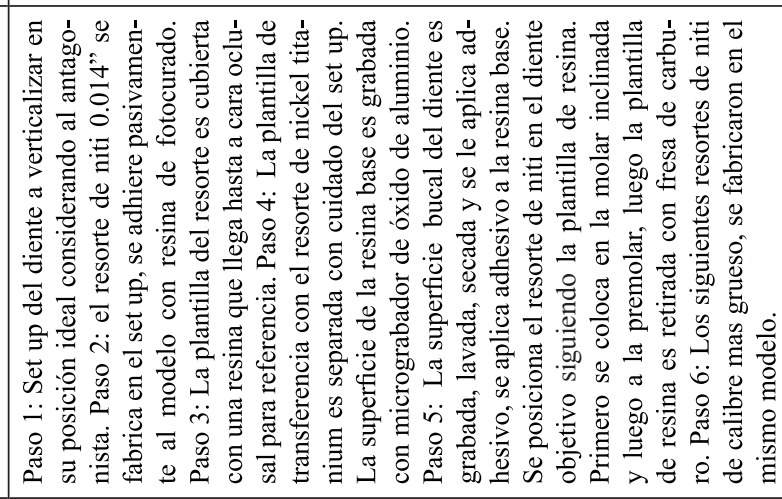 \\
\hline 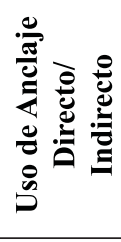 & 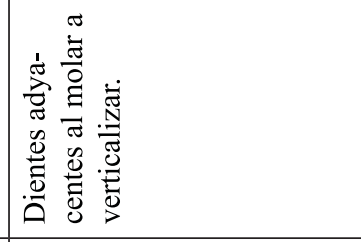 & 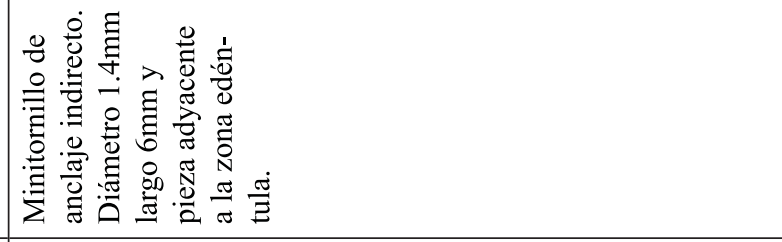 \\
\hline 㝵 & 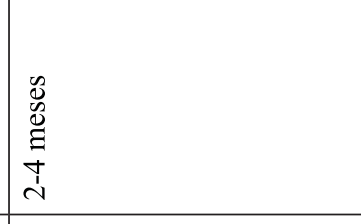 & 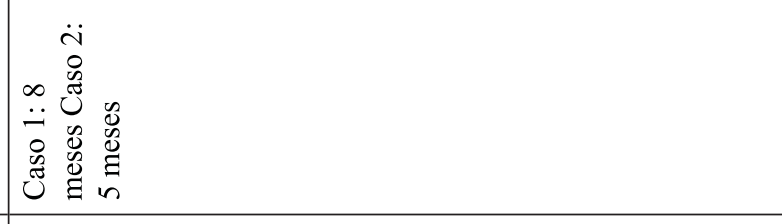 \\
\hline 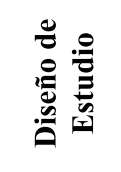 & 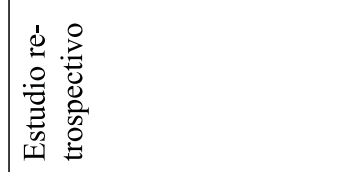 & 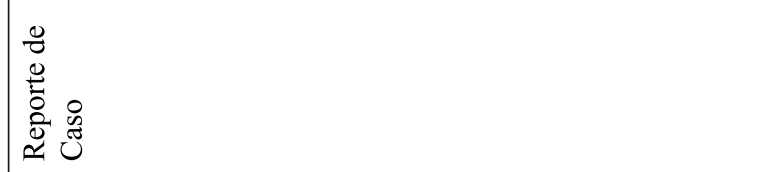 \\
\hline 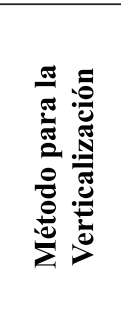 & 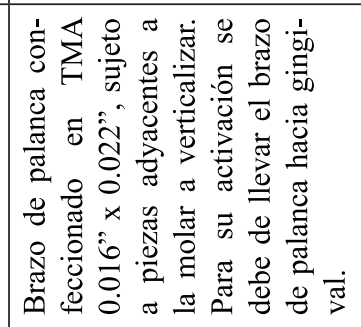 & 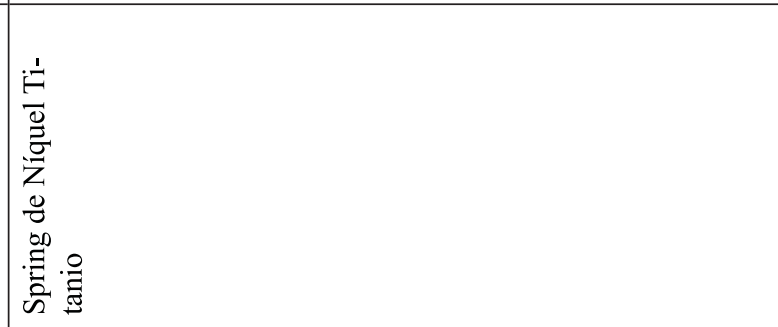 \\
\hline 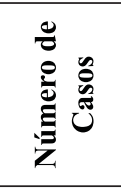 & 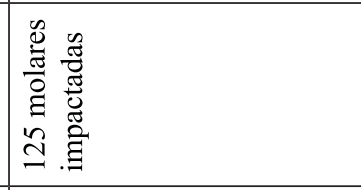 & $N$ \\
\hline 兽高 & 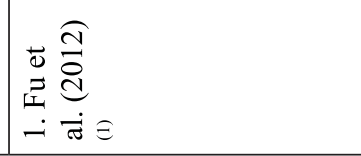 & 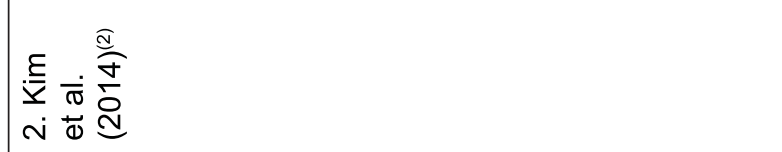 \\
\hline
\end{tabular}




\begin{tabular}{|c|c|c|}
\hline 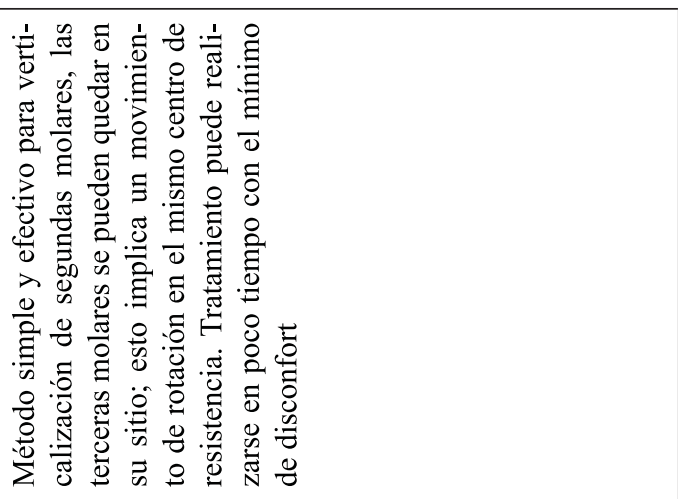 & 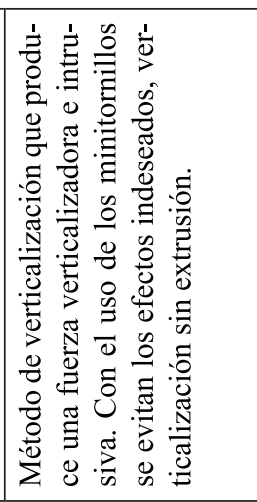 & 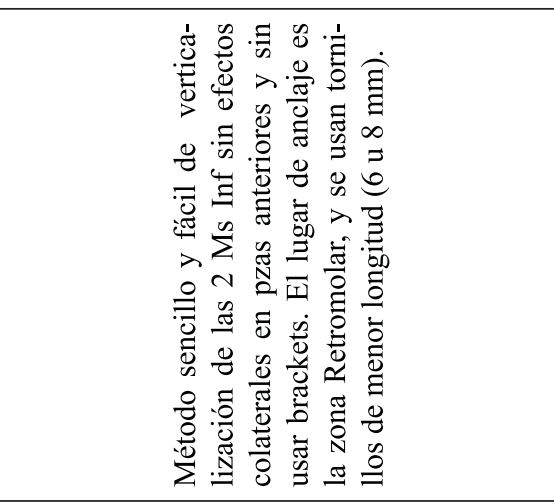 \\
\hline 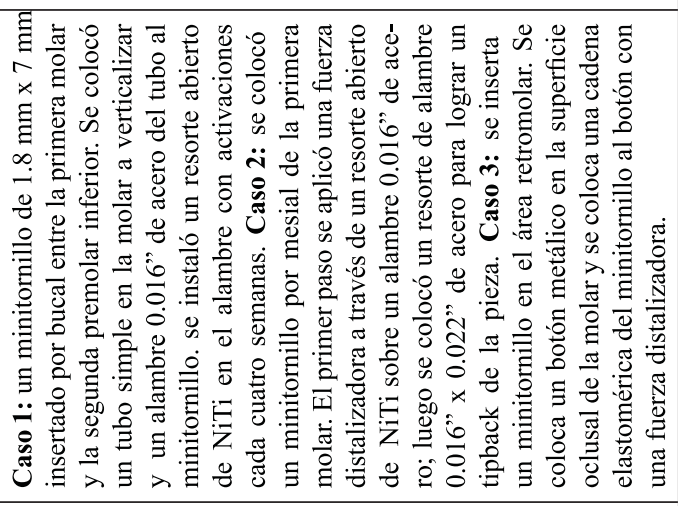 & 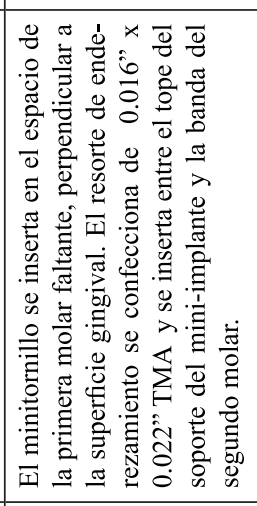 & 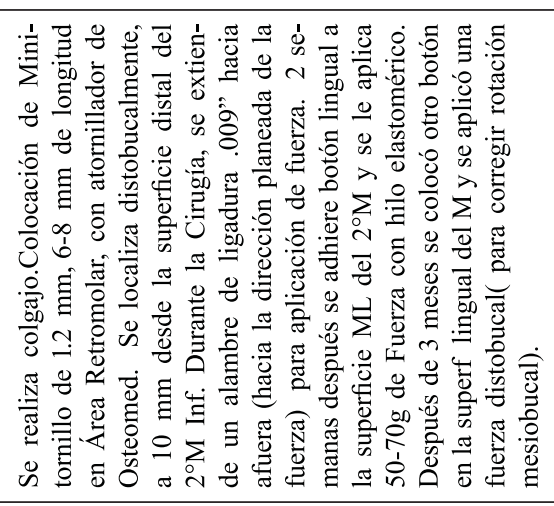 \\
\hline 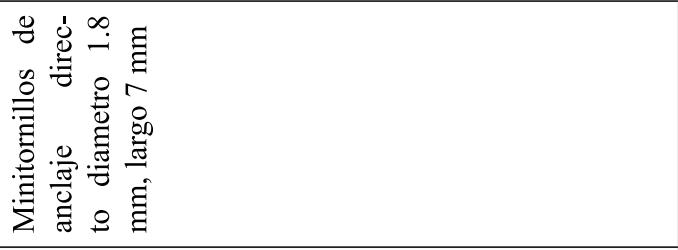 & 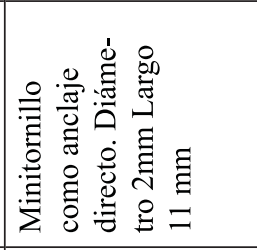 & 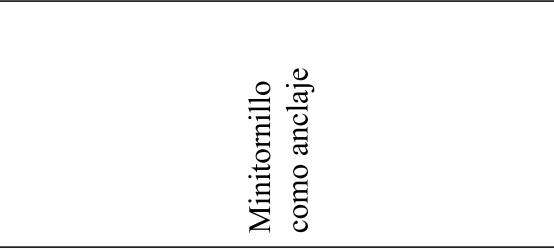 \\
\hline 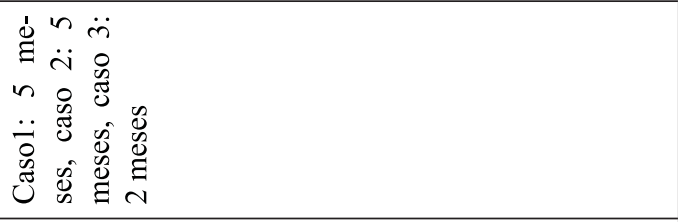 & 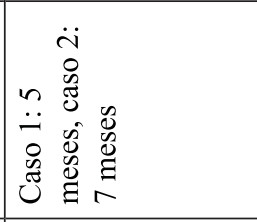 & 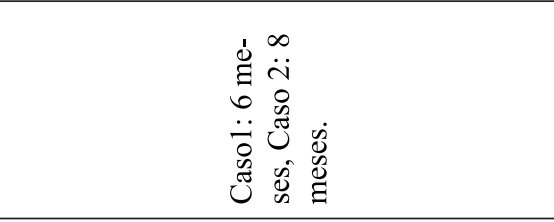 \\
\hline 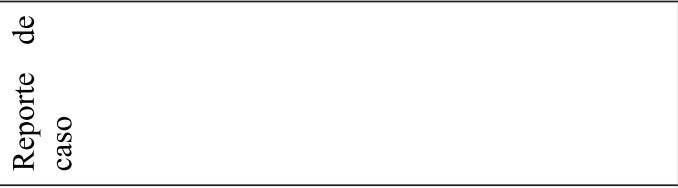 & 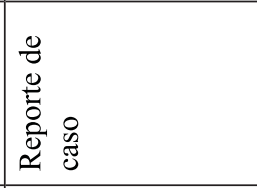 & 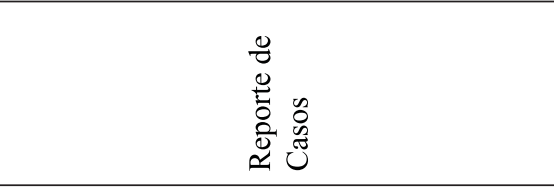 \\
\hline 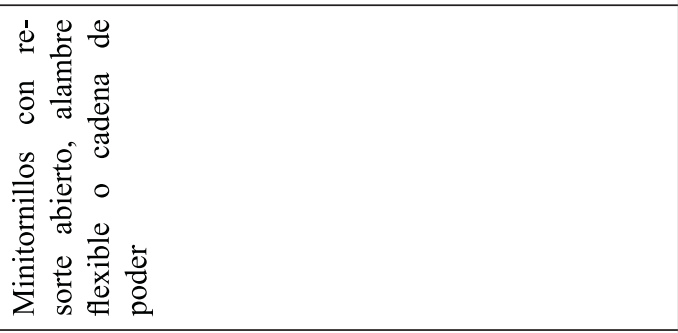 & 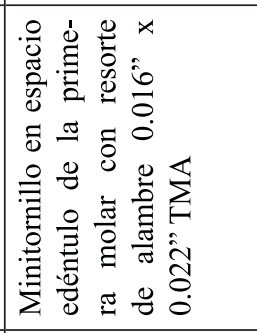 & 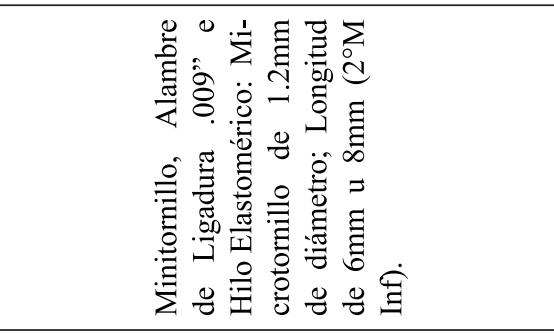 \\
\hline$m$ & N & $m$ \\
\hline 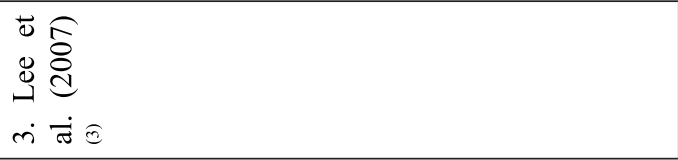 & 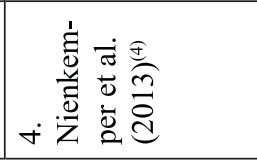 & 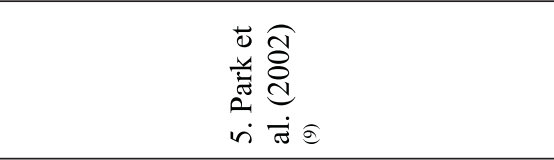 \\
\hline
\end{tabular}




\begin{tabular}{|c|c|}
\hline 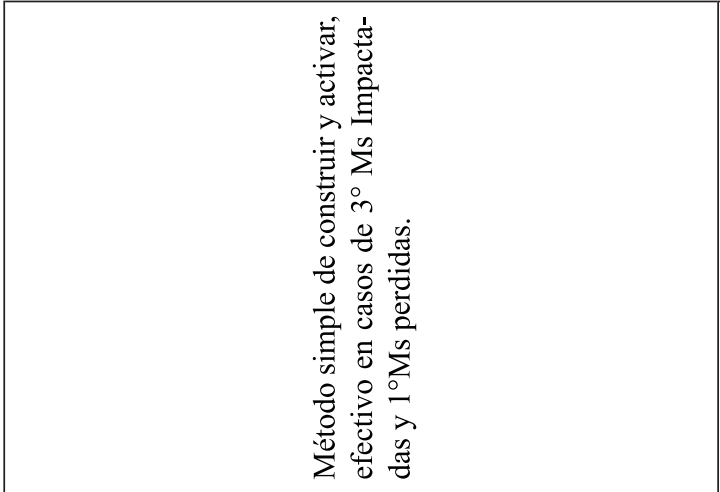 & 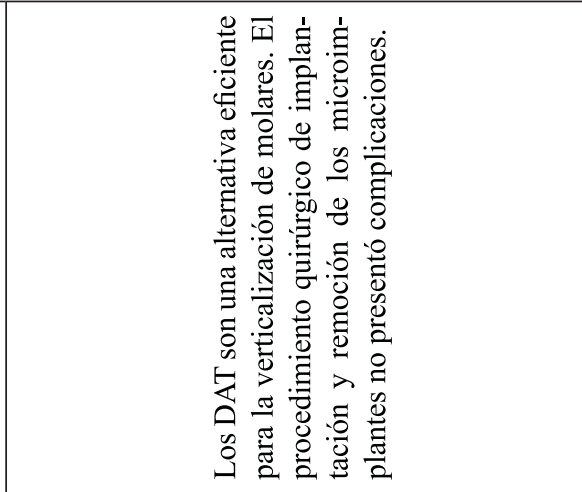 \\
\hline 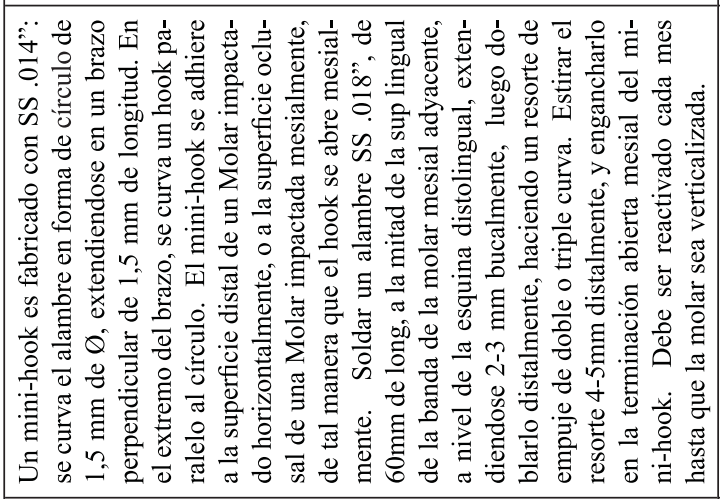 & 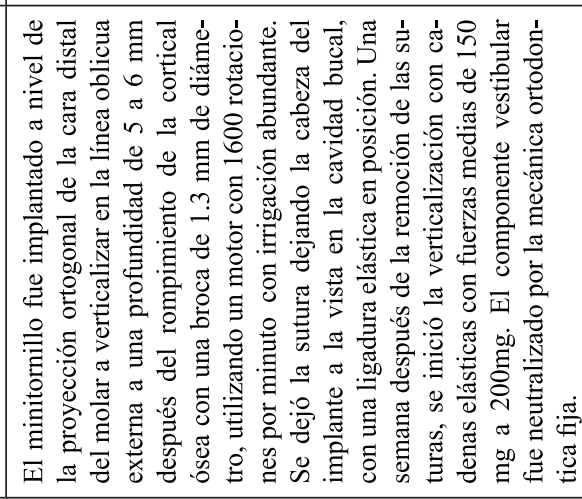 \\
\hline 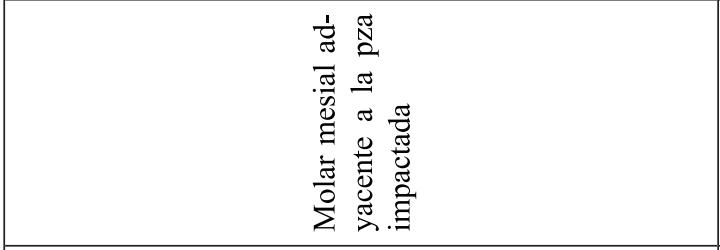 & 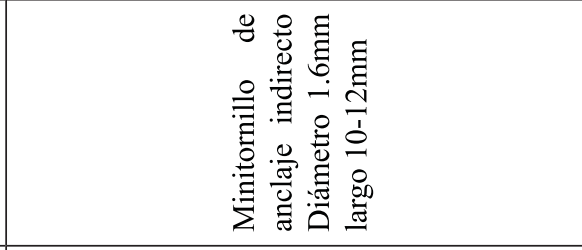 \\
\hline 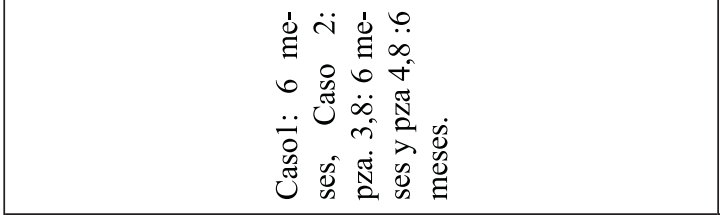 & 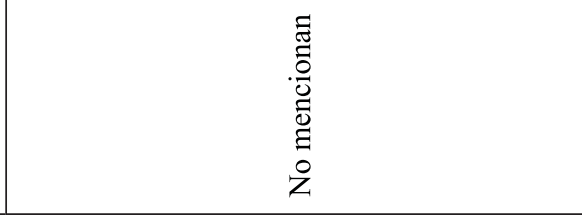 \\
\hline 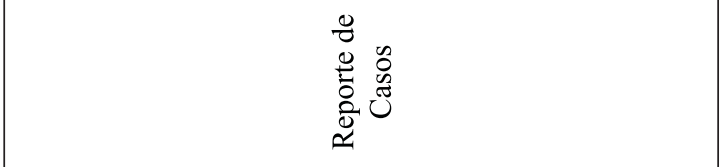 & 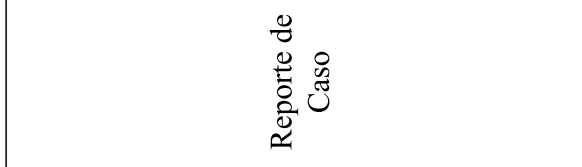 \\
\hline 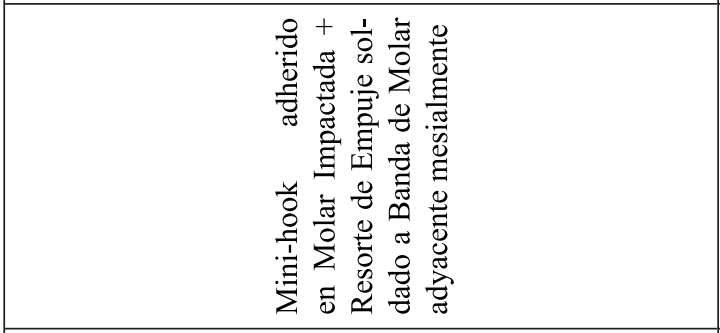 & 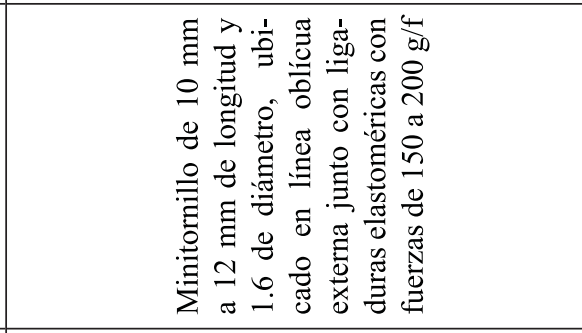 \\
\hline$\sim$ & $m$ \\
\hline 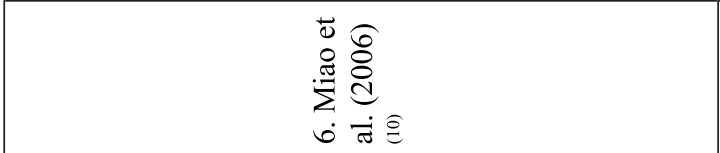 & 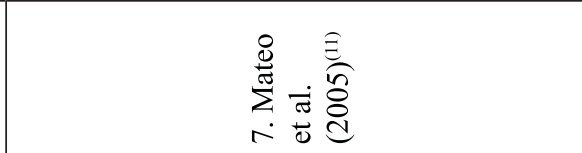 \\
\hline
\end{tabular}




\begin{tabular}{|c|c|c|c|c|c|}
\hline \multicolumn{3}{|c|}{ 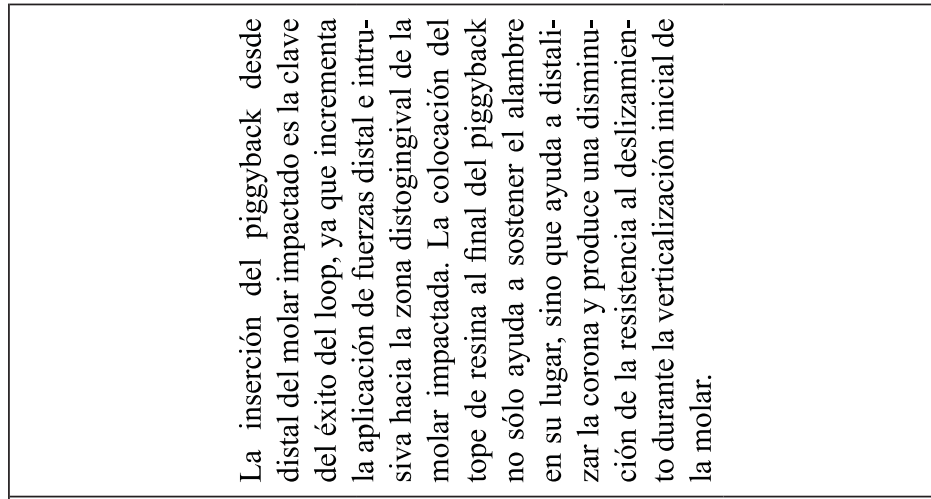 } & \multicolumn{3}{|c|}{ 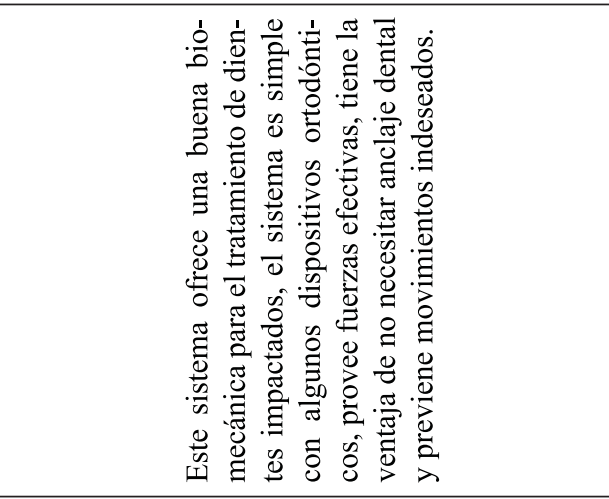 } \\
\hline 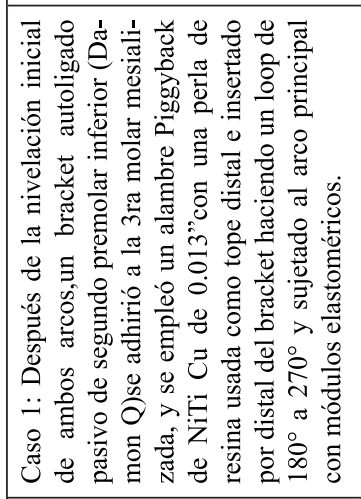 & 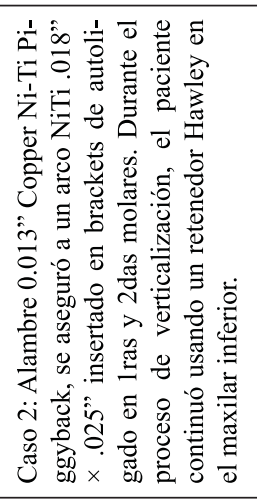 & 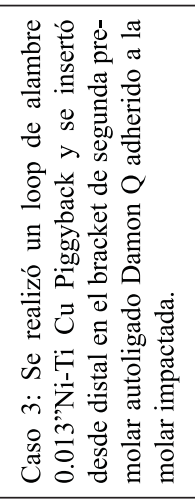 & 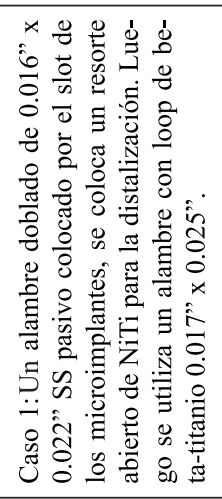 & 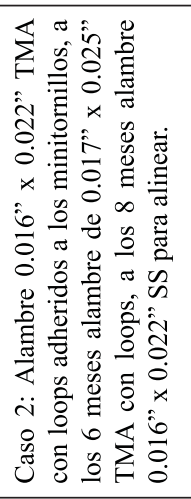 & 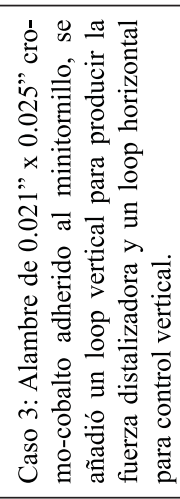 \\
\hline \multicolumn{3}{|c|}{ 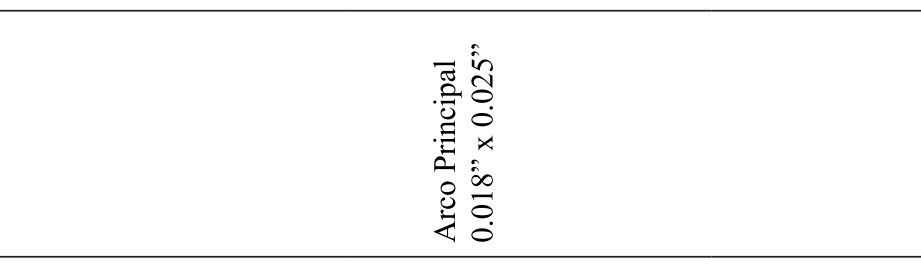 } & \multicolumn{3}{|c|}{ 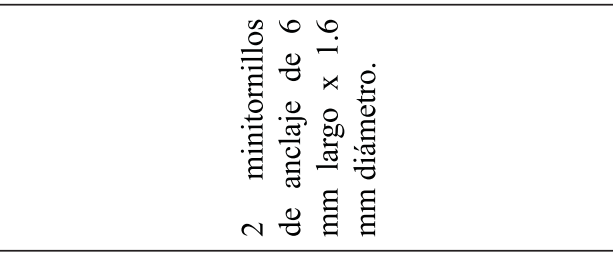 } \\
\hline 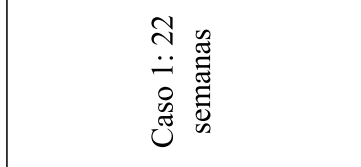 & 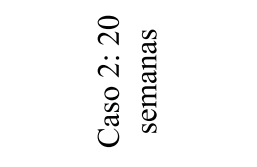 & 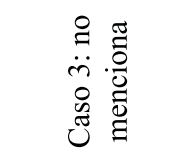 & 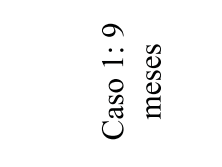 & 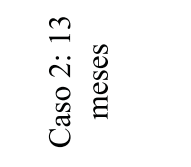 & 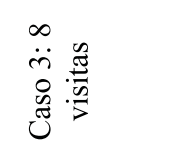 \\
\hline \multicolumn{3}{|c|}{ 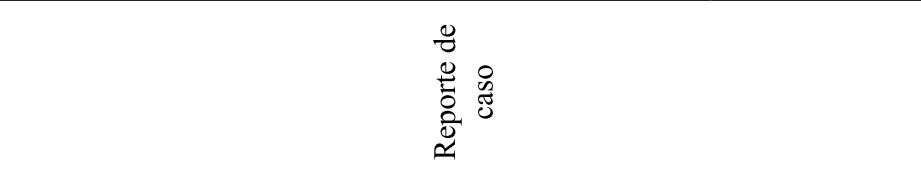 } & \multicolumn{3}{|c|}{ 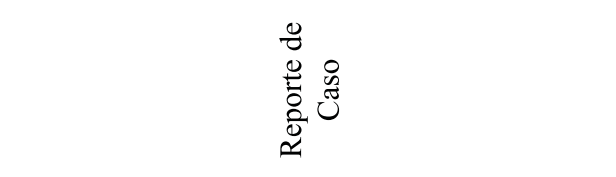 } \\
\hline \multicolumn{3}{|c|}{ 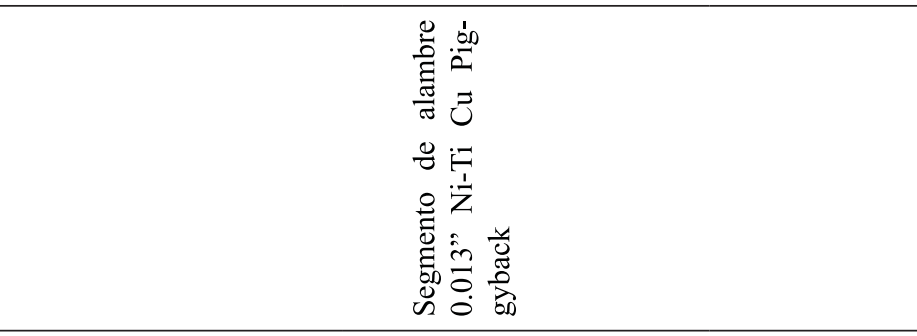 } & \multicolumn{3}{|c|}{ 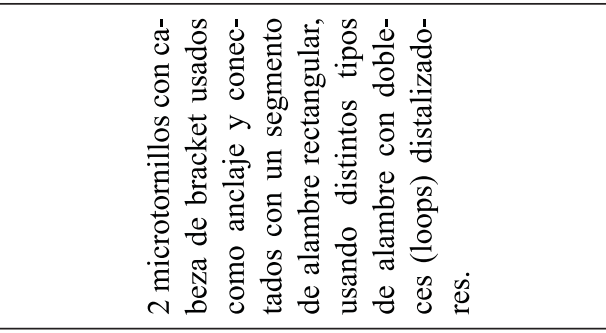 } \\
\hline \multicolumn{3}{|c|}{$n$} & \multicolumn{3}{|c|}{$m$} \\
\hline \multicolumn{3}{|c|}{ 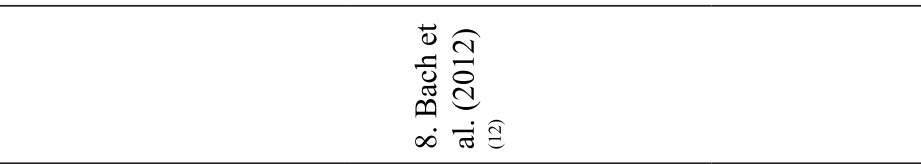 } & \multicolumn{3}{|c|}{ 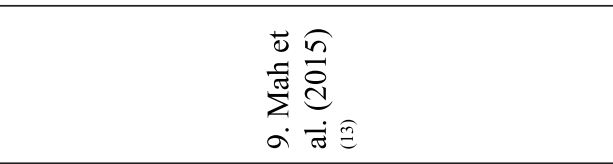 } \\
\hline
\end{tabular}




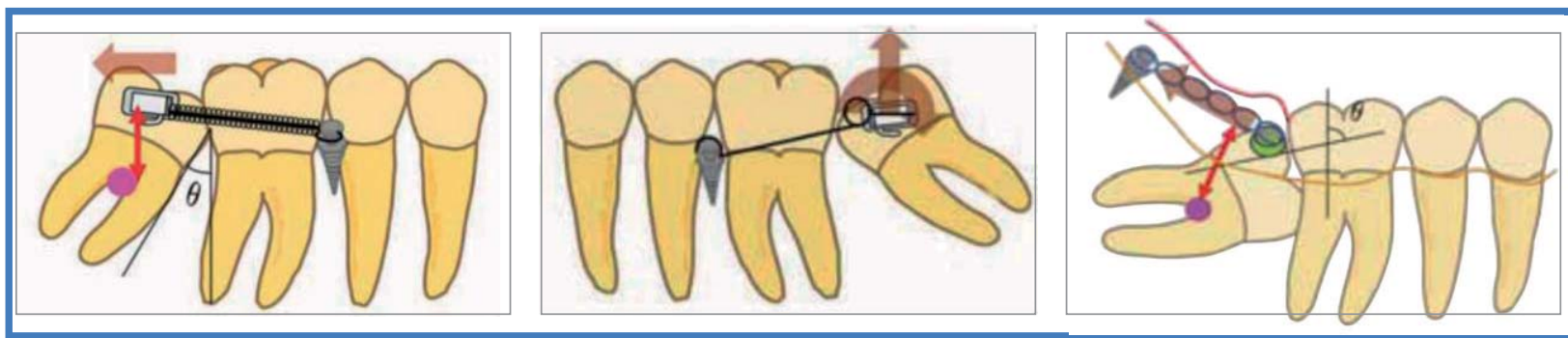

Figura 3. Lee et al. $(2007)^{3}$

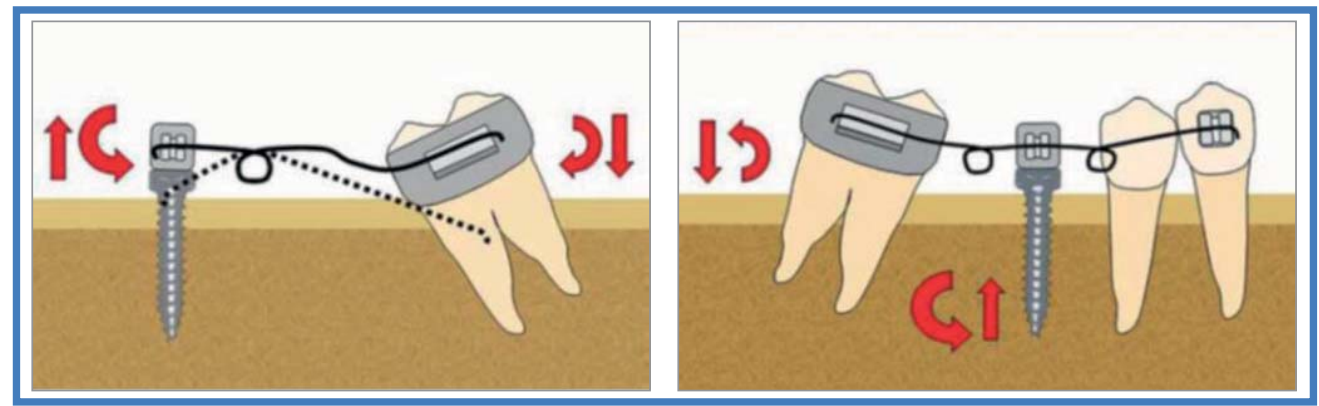

Figura 4. Nienkemper et al. (2013)4

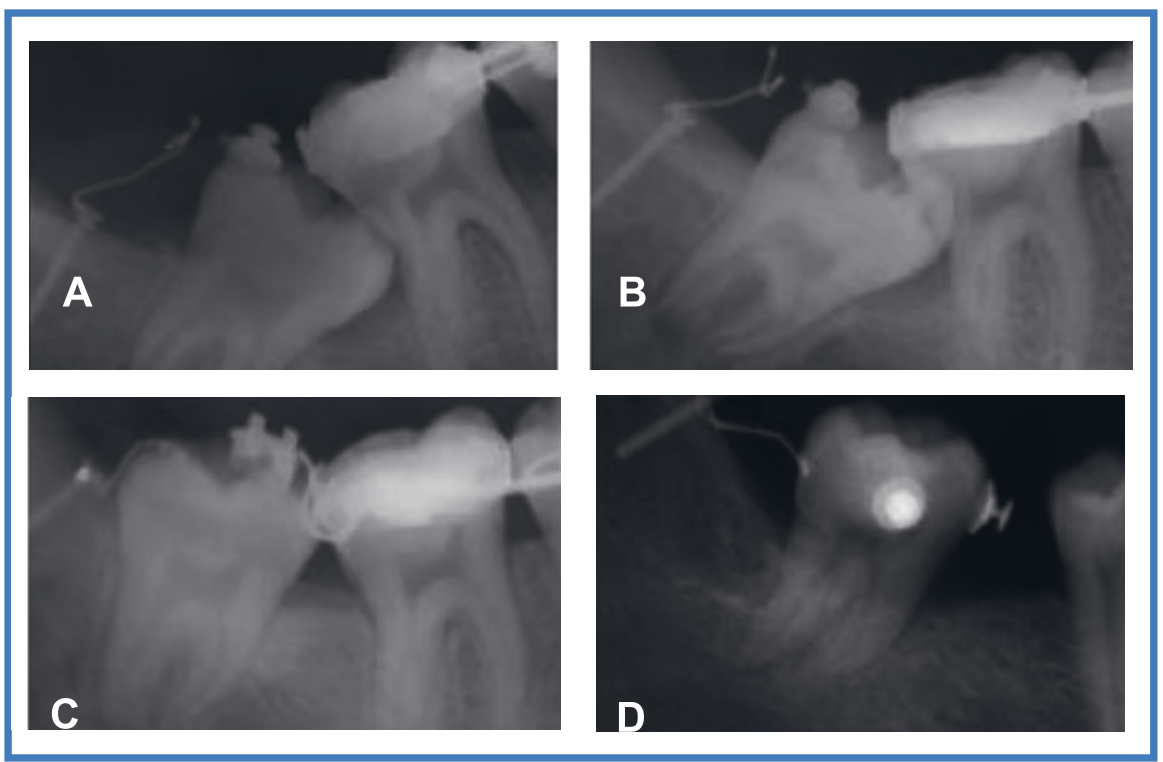

Figura 5. Park et al. (2002) ${ }^{9}$ 

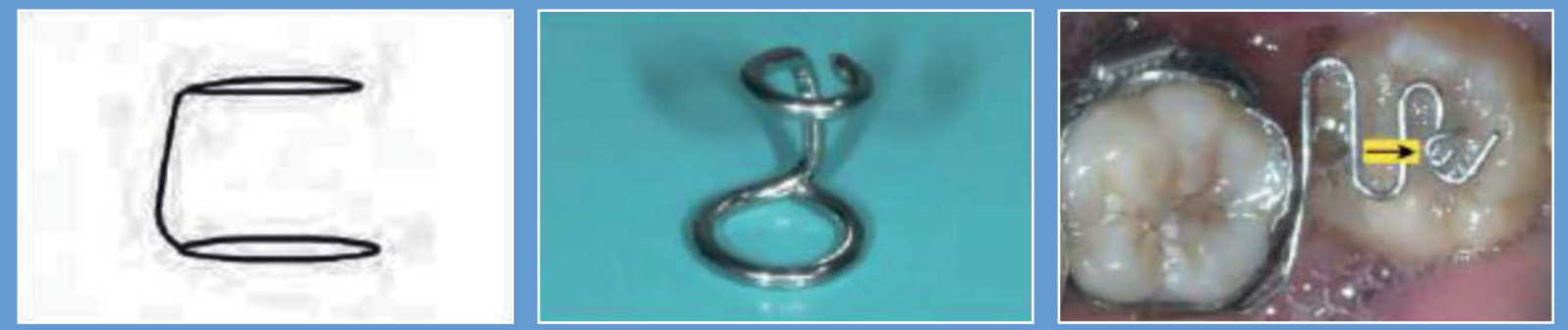

Fig. 1 Fabrication and bonding of mini-hook (arrow)
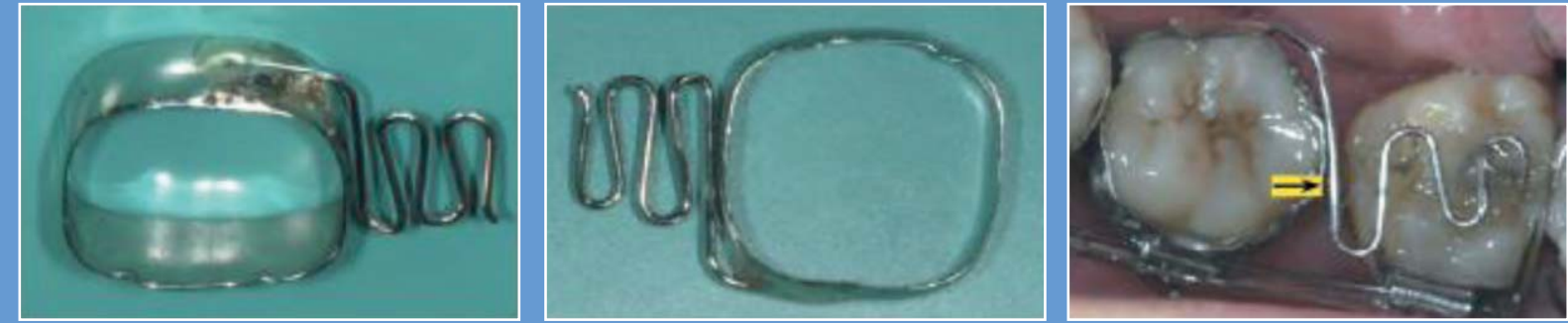

Fig. 1 Fabrication and placement of push-spring (arrow) to move crown of impacted molar distally and occlusally.

Figura 6. Miao et al. $(2006)^{10}$

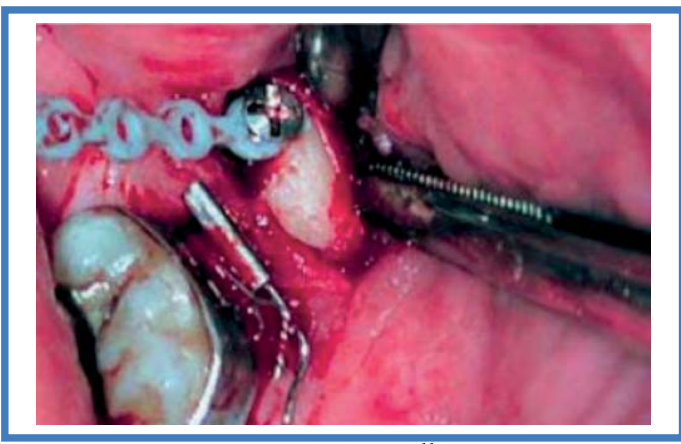

Figura 7. Matteo et al. $(2005)^{11}$
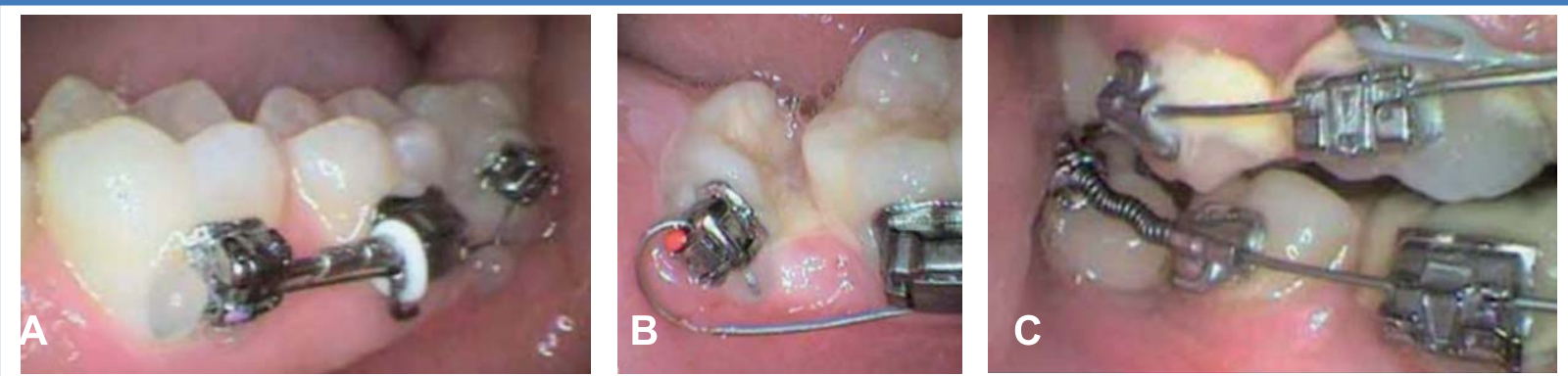

Figura 8. Bach et al. $(2012)^{12}$

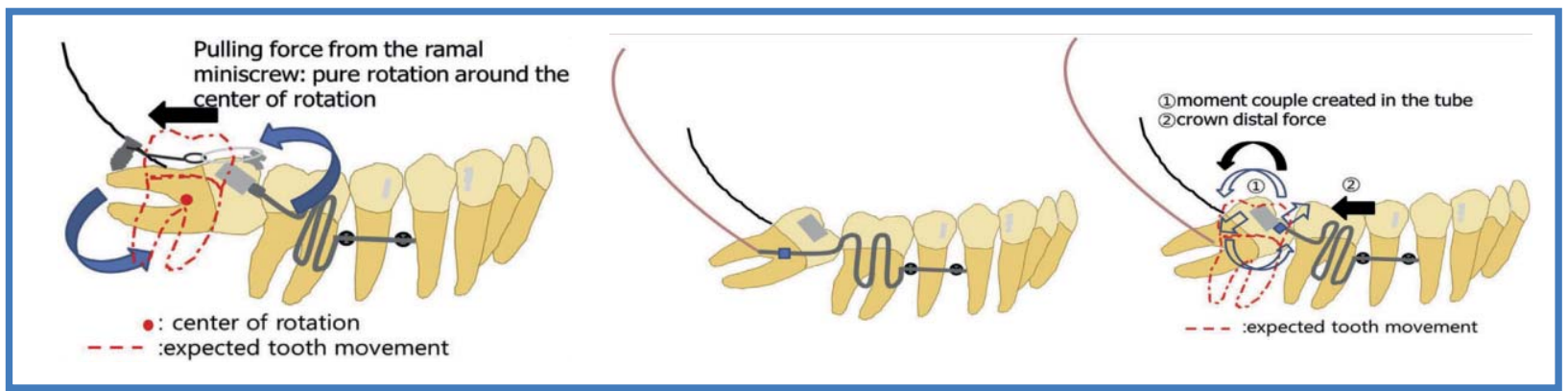

Figura 9. Mah et al. $(2015)^{13}$ 


\section{DISCUSIÓN}

Muchas veces en pacientes que han perdido primeras molares, las segundas han mostrado mesioangulación. La restauración de dientes ausentes en un paciente adulto es difícil cuando existen pilares inclinados que serán usados por el rehabilitador. ${ }^{5}$ Se han realizado diversas técnicas con el propósito de verticalizar molares impactadas severamente, ${ }^{6-8}$ requiriendo experiencia y paciencia del profesional. Muchas de ellas usan aparatos removibles necesitando la cooperación del paciente, sin embargo, la mayoría requiere exposición quirúrgica de la molar impactada. El tiempo de tratamiento puede extenderse debido a que toma más tiempo el mover molares impactados primero hacia distal y luego hacia oclusal, que lograr estos dos movimientos al mismo tiempo. ${ }^{7}$

Sólo nueve estudios cumplieron con los criterios de inclusión establecidos en esta revisión de casos. Esto remarca la escasez de estudios consistentes y la falta de evidencia científica para ayudar a los ortodoncistas a elegir la mejor opción para lograr la verticalización de molares. Sólo un estudio cuenta con un alto número de casos, ${ }^{1}$ por lo tanto, las comparaciones con los otros reportes, se realizaron de una manera limitada debido a las diferencias en selección y tamaño de la muestra.

Seis reportes de casos se realizaron con minitornillos de anclaje, siendo una alternativa eficiente para la verticalización de molares, sin efectos colaterales en otras piezas dentarias. ${ }^{2,4,6-9,11}$ Mientras que Miao et al. ${ }^{10}$ Usaron como anclaje una molar adyacente a la zona mesial de la pieza impactada, logrando verticalizar efectivamente la segunda y tercera molar inferior con un aparato fijo (minihook), adherido a la superficie de la pieza impactada, sin embargo no fueron evaluados los efectos indeseados en la molar usada como anclaje.

Fu et al. ${ }^{1}$ utilizaron un brazo de palanca confeccionado en TMA 0.016 " x 0.022 ", sujeto a piezas adyacentes a la molar a verticalizar, en 125 casos, reportando el resultado deseado en un tiempo menor de 2 a 4 meses, pero sin evaluar los efectos indeseados en las piezas adyacentes.
Bach $^{12}$ empleó un segmento de alambre Piggyback NiTi Cu 0.013" con una perla de resina usada como tope en distal que se colocaba hacia gingival del arco principal insertándolo por distal en un bracket de autoligado Damon adherido a la pieza impactada, haciendo un loop de $180^{\circ}$ a $270^{\circ}$ y sujetado al arco principal con módulos elastoméricos. Para este método se requirieron alinear y nivelar previamente, además se usa como anclaje las otras piezas dentarias, sin tomar en cuenta el posible efecto colateral en ellas.

Mah y et al. ${ }^{13}$ usaron 2 microtornillos con cabeza de bracket usados como anclaje y conectados con un segmento de alambre rectangular, para controlar tridimensionalmente la molar impactada, usando distintos tipos de alambre con dobleces (loops) distalizadores. En este estudio, se usaron tres casos para verticalizar segundas molares impactadas en pacientes que no requerían tratamiento ortodóntico adicional. Por lo cual mencionan como beneficio que no requieren anclaje en las piezas adyacentes ni generan efectos indeseables en ellas.

Así mismo, Kim et al. ${ }^{2}$ usaron minitornillos como anclaje indirecto, en este reporte de casos se realiza un set up en el laboratorio previo a la confección del verticalizador, generando tiempo de trabajo adicional para el ortodoncista. Lee et al, ${ }^{3}$ usaron minitornillos como anclaje directo realizando la verticalización con el uso de diversos elementos como resorte abierto de NiTi y cadenas de poder. En contraste, Nienkemper ${ }^{4}$ reportó 2 casos en los cuales usó minitornillos colocados verticalmente en la zona de la pieza ausente como anclaje directo usando resorte verticalizador de TMA rectangular, ya que éste genera un efecto verticalizador y una fuerza intrusiva, a la vez que aplica un momento significativo a la unidad de anclaje, lo cual es controlado con el uso del anclaje esquelético.

Park et al. ${ }^{9}$ colocaron minitornillos como anclaje directo, a distal de la pieza a verticalizar, utilizando fuerzas leves de 50-70 g, logrando la verticalización en un promedio de tiempo de 7 meses, 
haciendo uso de hilo elastomérico y alambre de ligadura 0,009 ", sin producir efectos colaterales en las piezas adyacentes.

Matteo et al. ${ }^{11}$ hacen uso de minitornillos como anclaje directo, aplicando fuerzas de 150 a $200 \mathrm{~g}$ con cadenas elastoméricas después de una semana del retiro de sutura, no aplicando carga inmediata en el implante.

La revisión de estos casos clínicos para verticalización de molares demuestra que, el uso de minitornillos como sistema de anclaje es el método más efectivo que reduce los efectos indeseables. No todos los reportes mencionan la cantidad de fuerza aplicada a la molar durante la verticalización, por lo que se recomienda ensayos clínicos posteriores para indicar la fuerza ideal que se necesita para lograr la verticalización de la pieza en los distintos sistemas aplicados sin efectos colaterales como reabsorción radicular o lesiones en el periodonto, además de demostrar cúal de estos sistemas es el más eficiente.

Los resultados del presente estudio apuntan a la evidencia en favor del uso de los minitornillos como sistema de anclaje para la verticalización de piezas posteriores en combinación con resorte abierto, alambre flexible, cadena de poder o hilo elastomérico, ya que no produce efectos colaterales en piezas anteriores o adyacentes y permite la verticalización de la pieza sin extrusión de ésta, además de lograrlo en un periodo de tiempo pro medio de 5 a 6 meses.

El tipo de anclaje más eficiente es el de minitornillos, ya que no produce efectos colaterales en piezas anteriores y permite la verticalización de la pieza sin extrusión. Sin embargo, se necesitan estudios comparativos de las diferentes técnicas de verticalización en los cuales se evalúen los efectos indeseados y el tiempo de obtención de resultados. Además, se requieren estudios de alta validez para presentar sólida evidencia para el sustento de los resultados de esta revisión de casos clínicos.

\section{REFERENCIAS BIBLIOGRÁFICAS}

1. Fu PS, Wang JC, Wu YM, Huang TK, Chen WC, Tseng YC y col. Impacted mandibular second molars. A retrospective study of prevalence and treatment outcome. Angle Orthod. 2012; 82(4): 670-5.

2. Kim M, Kim M, Chun YS. Molar uprighting by a nickel-titanium spring based on a setup model. Am J Orthod Dentofacial Orthop. 2014;146(1): 119-23.

3. Lee KJ, Park YC, Hwang WS, Seong EH. Uprighting Mandibular Second Molars With Direct Miniscrew anchorage. J Clin Orthod. 2007;41(10):627-35.

4. Nienkemper M, Pauls A, Ludwig B, Wilmes B, Drescher D. Preprosthetic molar uprighting using skeletal anchorage. J. Clin Orthod. 2013;47: 433-7.

5. Valverde R, Talavera CJ. Verticalizacion de molares-Preparación ortodóntica del paciente protésico. Rev Estomatol Herediana. 2005;15(2):155-60.

6. Shapira Y, Borell G, Nahlieli O, Kuftinec MM, Stom D. Uprighting mesially impacted mandibular permanent second molars. Angle Orthod. 1998; 68(2):173-8.

7. Kogod M, Kogod HS. Molar uprighting with the piggyback buccal sectional arch wire technique, Am. J. Orthod Dentofacial Orthop. 1991; 99:276-80.

8. Majourau, A, Norton LA. Uprighting impacted second molars with segmented springs, Am J Orthod Dentofacial Orthop. 1995; 107(3):235-8.

9. Park HS, Kyung HM, Sung JH. A simple method of molar uprighting with micro-implant anchorage. J. Clin. Orthod. 2002;36(10): 592-6.

10. Miao YQ, Zhong H. An uprighting appliance for im pacted mandibular second and third molars. J. Clin. Orthod. 2006;40(2):110-6.

11. Di Matteo R, Villa N, Sendyk W. Movimentação de molares inferiores ancorados em mini-parafusos. $\mathrm{R}$ Dental Press Ortodon Ortop. 2005;10(4):124-33.

12. Bach R. Non-surgical uprighting of exposed, mesially impacted lower molars. J.Clin.Orthod. 2012;46(8):480-5.

13. Mah S, Won P, Nam J, Kim E, Kang Y. Uprighting mesially impacted mandibular molars with 2 miniscrews. Am J Orthod Dentofacial Orthop. 2015;148:849-61.

14. Johnson E, Taylor RC. A surgical-orthodontic approach in uprighting impacted mandibular second molars. Am J Orthod. 1972 May;61(5):508-14.

15. Lai Y, Chu MP, Vijayaraghavan TV. Uprighting molars with twisted superelastic nickel titanium wires. J Clin Orthod. 2001;35(2):100-5.

16. Musilli M, Marciso M, Romanucci A, Grampone F. Molar uprighting with mini screws: comparison among different systems and relative biomechanical analysis. Progress in Orthod. 2010;11(2):166-73. 
17. Tunkay OC, Biggerstaff RH, Cutcliffe JC, Berkowitzz J. Molar Uprighting with T-Loop Springs. J Am Dent Assoc. 1980;100(6):863-6.

18. Sawicka M, Racka-Pilszaka B, Rosnowska-Mazurkiewicz A. Uprighting Partially Impacted Permanent Second Molars. Angle Orthod. 2007;77(1):148-54.

19. Roberts WW, Chacker FM, Burstone CJ. A segmental approach to mandibular molar uprighting. Am J Orthod Dentofacial Orthop. 1982;81(3):177-84.

20. Simon RL. Rationale and Practical Technique for uprighting mesially inclined molars. J Prosthet Dent. 1984; 52(2):256-9.

21. Martino F, Hernandez R. Uprighting of a semi-impacted mandibular second molar with microimplant anchorage. J World Fed Orthod. 2013;2(3):159-62.

22. Pogrel MA. The surgical uprighting of mandibular second molars. Am J Orthod Dentofacial Orthop. 1995; 108(2):180-3.

23. Rao J, Kumar G, Singh G, Alvi HA, Kumar L, Agrawal KK. Removable partial denture with molar uprighting spring: An innovative hybrid appliance. J Prosthodont Res. 2013; 22(6):495-500.

24. Verma S, Kumar Gupta D, Singh G, Mittal S. A customized appliance for molar uprighting and space regaining. J Clin Orthod. 2015 Jul;49(7):452-4.

25. Fernandes AFC, Fernandes J, Quintao C, Mendes A. Uprighting an impacted Second Molar with a spring anchored to a composite support. J Clin Orthod. 2008; 42(5):301-2.

26. Park HS, Kwon OW, Sung JH. Uprighting Second Molars with Micro-Implant Anchorage. J Clin Orthod. 2004; 38(2):100-3.

27. Bondemark L. A comparative analysis of distal maxillary molar movement produced by a new lingual intra-arch Ni-Ti coil appliance and a magnetic appliance. Eur J Orthod. 2000;22(6):683-95.

28. Janson M, Janson R, Ferreira PM. Tratamento Interdiciplinar I: Considerações Clínicas e Biológicas na Verticalização de Molares. R Dental Press Ortodon Ortop Facial. 2001;6(3):1-18.

29. Sakima T, Martins LP, Sakima MT, Terata HH, Kawakami RY,Ozawa TO. Mechanical alternatives for molars uprighting. Force systems released by appliances. Rev Dent Press Ortodon Ortop Maxilar. 1999;4(1):79-100.

30. Allgayer S, Platcheck D, Vargas IA, Drumond Loro RC. Mini-implants: mechanical resource for molars uprighting. Dental Press J. Orthod. 2013;18(1):134-42.

31. Raghav S, Vinod P, Shashikala KV. The Neoslider appliance for uprighting mesially impacted mandibular second molars. J Clin Orthod. 2013;47(9):553-7.

32. Khouw FE, Norton LA. The mechanism of fixed molar uprighting appliances. J Prosthet Dent.1972;27(4):381-9.

33. Lau CK, Whang CZ, Bister D. Orthodontic uprighting of severely impacted mandibular second molars. Am J Orthod Dentofac Orthop. 2013;143(1):116-24.

34. Miyahira YI, Maltagliati LA, Siqueira DF, Romano R. Miniplates as skeletal anchorage for treating mandibular second molar impactions. Am J Orthod Dentofacial Orthop. 2008;134(1):145-8.

35. Ohura R, Kuroda S, Takahashi T, Tomita Y, Tanaka E. Efficient usage of implant anchorage to treat overerupted maxillary first molar and mesially inclined mandibular molars. Am J Orthod Dentofacial Orthop. 2011;139(1):113-22.

36. Majourau A, Norton LA. Uprighting impacted second molars with segmented springs. Am J Orthod Dentofac Orthop. 1995;107(3):235-8.

37. Henns RJ. Uprighting Impacted Mandibular Second Molars. Angle Orthod. 1975;45(4):314-5.

38. Shellhart WC, Moawad M, Lake P. Case Report: Implants as anchorage for molar uprighting and intrusion. Angle Orthod. 1966;66(3):169-72.

39. Ferrazzini G. Uprighting of a deeply impacted mandibular second molar. Am J Orthod Dentofac Orthop. 1989;96(2):168-71.

40. Oesterle WC; Uprighting molars without extrusion. JADA.1999;130:381-85.

41. Kojima Y, Mizuno T, Fukui H. A numerical Simulation of tooth movement produced by molar uprighting spring. Am J Orthod Dentofac Orthop. 2007; 132(5):630-8.

42. De Faria R. Utilizacao de Ancoragem esqueletica indireta para verticalizacao de molares inferiores. Dental Press J Orthod. 2009; 8: 63-8.

43. Totti AMG, Ferreira ER, Bernardes LAA, Ferreira JTL, Matsumoto MAN, Romano FL. Verticalizacao de Segundos Molares inferiores com Arco Lingual Modificado: Relato de Caso. Rev Odontol Univ Cidade de Sao Paulo. 2010; 22 (3): 254-62.

44. Kravitz ND. Surgical Uprighting of Lower Second Molars. J Clin Orthod. 2016; 50(1): 33-40.

45. De Oliveira AC. Miniscrew- supported coil spring for molar uprighting: description. Dental Press Journal of Orthodontics 2013; 18(1): 134-42.

46. Tsay TP. A "Renewable" Archwire for uprighting impacted lower second molars. J Clin Orthod 2016; 50(4):254-7.

47. Giancotti A, Arcuri C, Barlattani A. Treatment of ectopic mandibular second molar with titanium miniscrews. Am J Orthod Dentofacial Orthop. 2004; 126: 113-17.

\section{CORRESPONDENCIA:}

Silvia Fuentes Valera

email:silvinja@hotmail.com 